\title{
Desmoid Tumor of the Chest Wall Mimicking Recurrent Breast Cancer: Multimodality Imaging Findings
}

\author{
Kyeong A Choi, ${ }^{1}$ and Yeong Yi An ${ }^{1, *}$ \\ ${ }^{1}$ Department of Radiology, St. Vincent's Hospital, College of Medicine, The Catholic University of Korea, Seoul, Republic of Korea \\ "Corresponding author: Yeong Yi An, Department of Radiology, St. Vincent's Hospital, College of Medicine, The Catholic University of Korea, Seoul, Republic of Korea. Tel: \\ +82-312498495, Fax: +82-312475713, E-mail: didi97@catholic.ac.kr
}

Received 2015 July 22; Revised 2015 September 04; Accepted 2015 September 14.

\begin{abstract}
Desmoid tumor of breast is a rare benign, locally aggressive tumor with a high recurrence rate. It has been associated with scar from previous breast surgery or trauma. Especially in breast cancer patients with previous operation history, it may simulate recurrent breast cancer clinically and radiologically. We presented multimodality imaging findings (ultrasound, computed tomography, magnetic resonance imaging and positron emission tomography/computed tomography) of chest wall desmoid tumor mimicking recurrent breast cancer in a 38-year-old patient with a history of left modified mastectomy. The desmoid tumor is a rare benign tumor that should be considered in the differential diagnosis of malignant local tumor recurrence after breast cancer operation. Biopsy was required for accurate diagnosis and wide local excision was its appropriate surgical management.
\end{abstract}

Keywords: Desmoid Tumor, Recurrent Breast Cancer, Modified Radical Mastectomy, Local Tumor Recurrence

\section{Introduction}

Desmoid tumor of breast is a rare benign tumor (1). While it lacks a metastatic potential, it may have an aggressive feature to infiltrate surrounding structures with a high recurrence rate after resection. Several case series describing its clinical, radiological and pathological features have been published. It can simulate malignancy clinically and radiologically and result in inappropriate tests and treatment including surgical procedures. It has been associated with scar tissue related to breast surgery or trauma, pregnancy, implants and familial adenomatous polyposis (1). Nevertheless, there were only a few case reports of desmoid tumor mimicking tumor recurrence after breast cancer surgery (2-5).

We presented a case of desmoid tumor with recurrence in a 38-year-old female with a history of left-sided breast cancer and left modified radical mastectomy. We described ultrasound (US), computed tomography (CT), magnetic resonance imaging (MRI) and positron emission tomography/computed tomography (PET/CT) appearances of this tumor and discussed the importance of early and appropriated diagnosis and its treatment.

\section{Case Presentation}

A 38-year-old woman had undergone modified radical mastectomy for an invasive ductal carcinoma of the left breast in April 2009. Histopathological examination revealed a $1.0 \times 0.7 \mathrm{~cm}$ invasive ductal carcinoma of histological grade 3 and all twenty resected axillary lymph nodes had negative results for carcinoma. Immunohistochemistry (IHC) staining showed that the left breast cancer had negative result for estrogen receptor (ER), progesterone receptor (PR), human epidermal growth factor receptor2 (HER-2) and p53. This patient was under postoperative imaging follow-up every 6 months and she was doing fine until November 2011, when she got a vague discomfort and palpable mass at left chest wall. On US, a huge heterogeneous hypoechoic mass was detected in the left axilla extending to the chest wall (Figure 1A). On chest CT, a $10 \times 4 \mathrm{~cm}$ irregular hypodense mass on the left lateral chest wall beneath the mastectomy site was noted. The mass showed an intense peripheral enhancement without adjacent bone destruction (Figure 1B). PET/CT examination revealed a large chest wall mass with moderate fluorodeoxyglucose (FDG) uptake (SUV $\mathrm{max}_{\max }$ of 3.1), suggestive of a malignant process rather than a benign process (Figure $1 C$ ). A focal mild FDG uptake ( $\mathrm{SUV}_{\max }$ of 2.1) was demonstrated in the enlarged left axillary lymph node.

Previous postoperative follow-up examinations were reviewed retrospectively. On the postoperative follow-up US conducted on May 2011 (Figure 1D), an about $3.5 \mathrm{~cm}$ illdefined hypoechoic area at the previous axillary dissection site was shown but discounted as postoperative scar and 

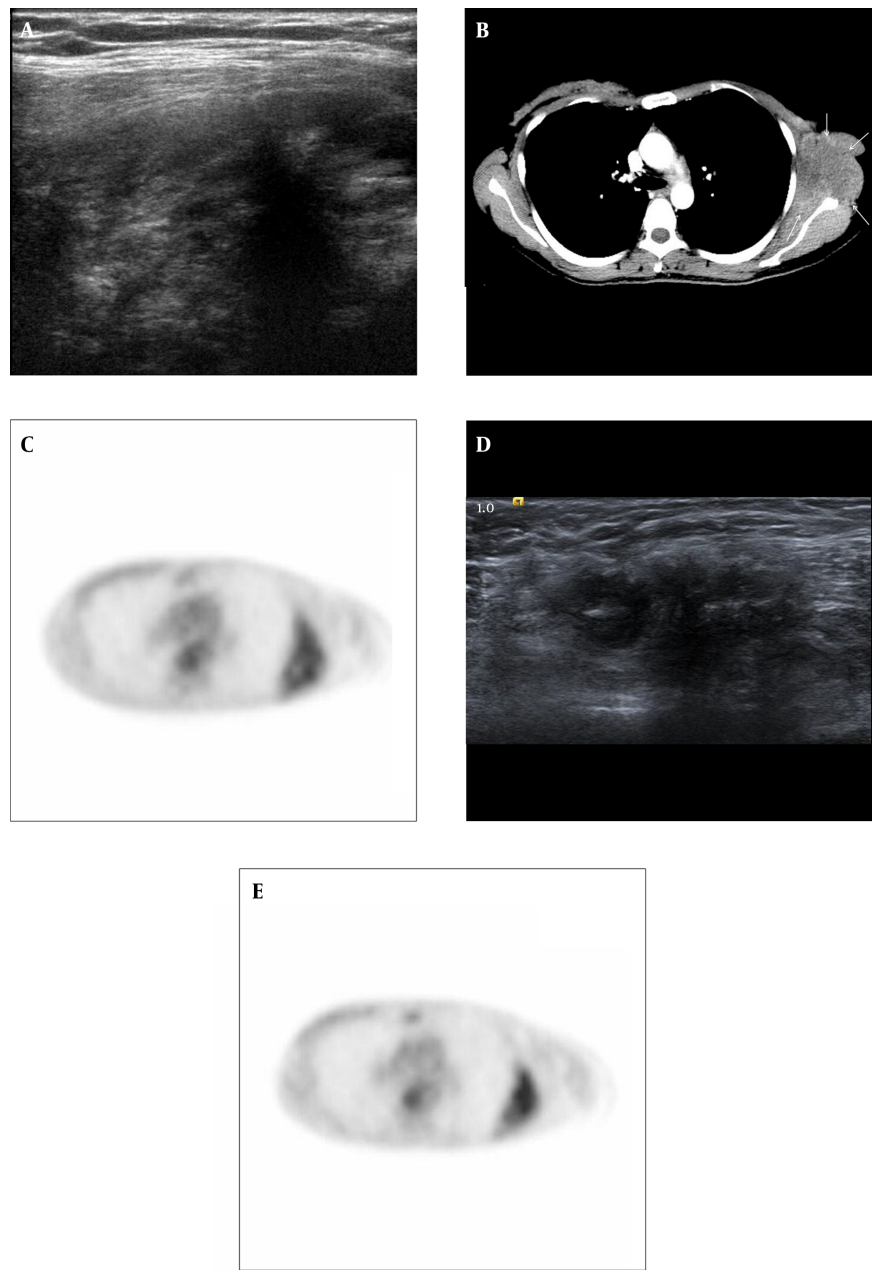

Figure 1. A 38-year-old woman with a history of left sided breast cancer treated with modified radical mastectomy underwent ultrasonography for further evaluation of palpable left chest wall mass. A, Breast US on November 2011. US showed a huge infiltrative heterogeneous hypoechoic mass at previous lymph node dissection site of left axilla, extending into left chest wall. B, Chest CT on November 2011; Chest CT showed a 10-cm irregular hypodense chest wall mass with intense peripheral enhancement beneath mastectomy site. There was no evidence of adjacent bone destruction. C, PET/CT on June 2011; PET/CT showed mild FDG uptake of a SUV ${ }_{\text {max }}$ of 3.1 at left lateral chest wall. D, US on May 2011 and E, PET/CT on June 2011; D and E on the postoperative follow-up examination undertaken 6 months before, this mass was misinterpreted as postoperative scar and fat necrosis. D, US showed a $3.5 \mathrm{~cm}$ irregular hypoechoic lesion at left axilla and E, PET/CT showed mild FDG uptake of a SUV $\max$ of 2.6 at left lateral chest wall. The size and FDG uptake of this lesion was increased 6 months later $\left(3.5 \mathrm{~cm}\right.$ to $10 \mathrm{~cm}$ in size, 2.6 to 3.1 in SUV $_{\max }$ ).

fat necrosis. As compared with previous examinations, the tumor increased in size after 6 months. On the postoperative follow-up PET/CT performed on June 2011, a mild FDG uptake $\left(S V_{\max }\right.$ of 2.6$)$ in the left axillary region was also misinterpreted as a postoperative change (Figure 1E).

A non-enhanced shoulder MRI (Figure 2A and 2B) and dynamic contrast enhanced breast MRI (Figure 2C) examinations were performed for the diagnosis and evaluation of the tumor. Huge infiltrative chest wall mass revealed isointense signal intensity to muscle on T1-weighted images (Figure 2A) and heterogeneous hyperintense signal intensity with several hypodense foci on T2-weighted images (Figure 2B). The mass involved in teres minor and major muscles, latissimus dorsi muscle, serratus anterior muscle, subscapularis muscle, focally infraspinatus muscle and chest wall muscles. After the contrast agent administration, the mass showed an intense heterogeneous enhancement (Figure 2C). The tumor demonstrated a perineural and vascular infiltration of brachial vessels. Also an enlarged left axillary lymph node was demonstrated suggesting the possibility of a metastatic lymphadenopathy. MRI findings suggested an extensive recurrent breast cancer or a primary malignant soft tissue tumor such as a sarcoma, desmoid tumor and malignant fibrous histiocytoma.

The patient subsequently underwent a US guided 

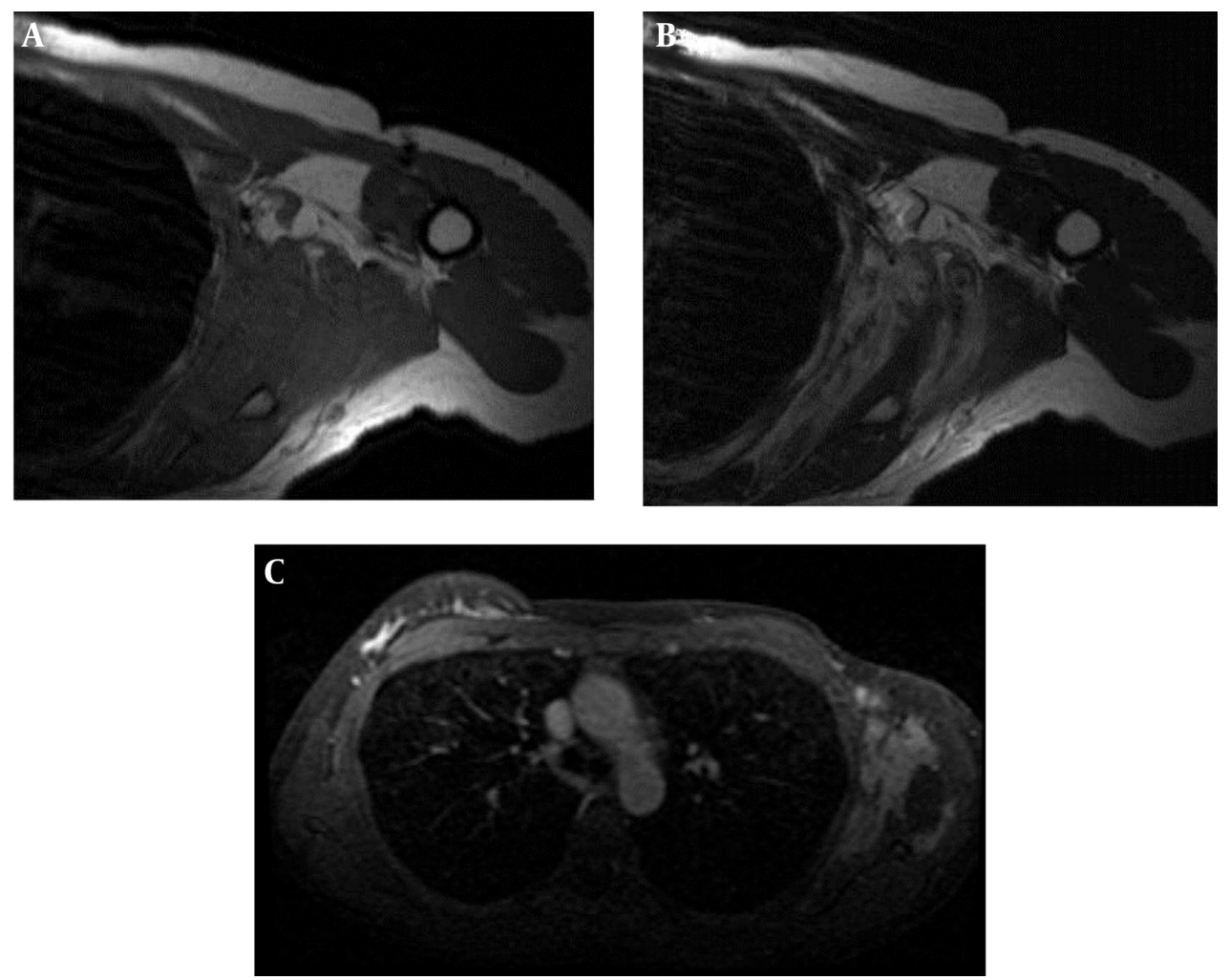

Figure 2. A 38-year-old woman with a history of left sided breast cancer treated with modified radical mastectomy underwent MRI of the chest wall. A, MRI T1 and B MRI T2; on non-enhanced left shoulder MRI, huge infiltrative soft tissue mass was isointense to muscle on axial T1-weighted images (A) and heterogeneous hyperintense with several hyposignal intensity foci on axial T2-weighted images (B). The mass had continuity with teres minor and major, latissimus dorsi, serratus anterior, subscapularis, focally infraspinatus and chest wall muscles. C, T1 Contrast enhanced MRI: after contrast administration, the mass showed an intense heterogeneous enhancement.

biopsy of the chest wall mass that revealed a spindle cell proliferation composed of fibroblastic cells. The possibility of benign mesenchymal neoplasm cannot be completely excluded. Therefore, wide excision with partial scapulectomy was performed for ultimate diagnosis. The final diagnosis was confirmed as desmoid tumor (Figure $3 \mathrm{~A})$. The immunohistochemical staining for $\beta$-catenin had positive result, which solidified the diagnosis (Figure 3B).

\section{Discussion}

Desmoid tumors are rare, accounting for $0.3 \%$ of all solid tumors (1). They are histologically benign, but local aggressive and tend to have high recurrence rates from $24 \%$ to $77 \%$ after 10 years (6). Desmoid tumors are characterized by fibroblastic proliferation from the connective tissue of the muscle and its overlying fascia aponeurosis. The most common extra-abdominal sites are shoulder, chest wall and inguinal region, although $30-50 \%$ of desmoids arise in the abdominal cavity. The etiology of desmoid tumors is poorly understood yet. They may also occur sporad- ically. Also an initial desmoid tumor was reported in a patient with a familial adenomatous polyposis syndrome, so called Gardner's syndrome (7). Up to $30 \%$ of patients have a trauma history (8) such as a breast reduction or breast augmentation with silicon or saline implants (9-11). It is supposed that desmoid tumors originate in the fibrous capsule of the implant. Although it may occur at any age, several studies have shown that women of reproductive age are afflicted more often than other groups. In addition, estrogen dominance, as during pregnancy, may be a significant predisposition for the development of a desmoid tu$\operatorname{mor}(1,12)$.

It has been reported in the literature that chest wall desmoid tumors might stimulate breast cancer clinically and radiologically. Only a few cases have been reported where desmoid tumors mimicking a tumor recurrence in breast cancer patients after conserving operation (2-5). For the patient with a history of breast cancer and operation, it is very confusing to provide a differential diagnosis other than recurrent breast cancer such as postoperative change or soft tissue tumor of the chest wall. 

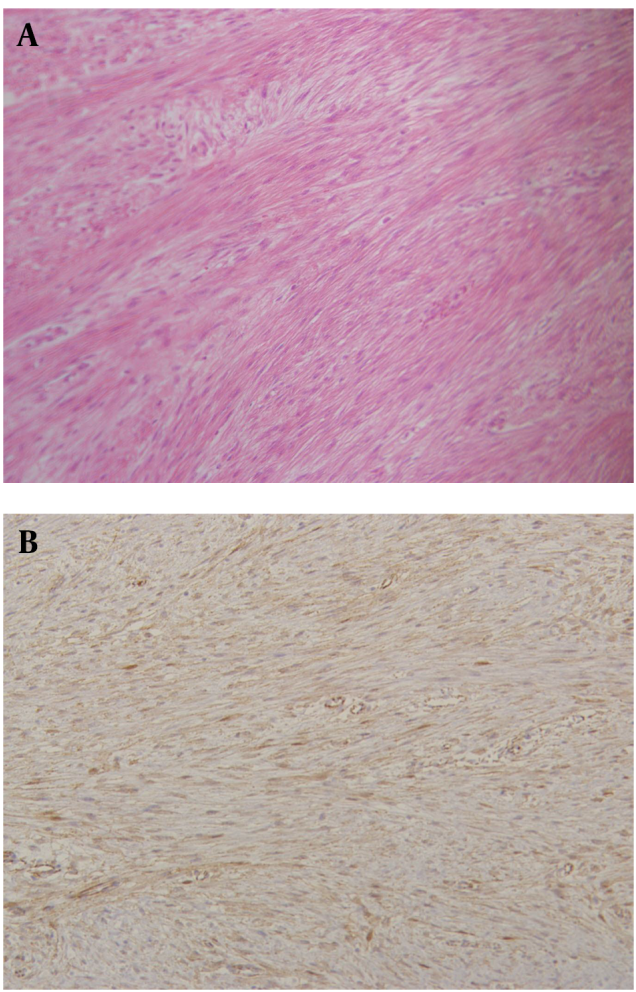

Figure 3. Histological specimen with hematoxylin and eosin (A) and beta-catenin staining (B). The tumor is composed of collagen fibers and spindle cells that stain positive for beta-catenin, consistent with a desmoid tumor

On physical examination, desmoid tumors present as a palpable hard mass, which might mimic malignant lesions. A dimpling or retraction of the skin may be found as the presented mass adheres to the chest wall (13). In mammography, desmoid tumor presents as an irregular, spiculated mass, which raises suspicion for malignancy. In a prior study of Neuman HB et al. (5), known desmoid tumors were visible in radiography in only a third of cases. US findings of desmoid tumors are an irregular spiculated or microlobulated, hypoechoic mass with straightening and tethering to the Cooper's ligaments, which simulates malignancy (14). CT findings of these lesions are variable and depend on the tumor composition, including the present collagen content and amount of solid or necrotic tissue. Lesions with a higher solid tissue component have a greater attenuation and enhancement. On MRI, these lesions have a similar signal to muscles on T1-weighted images, with a very high signal on T2-weighted images (15). Low signal areas are also seen on T1- and T2 weighted images, which are thought to be hypocellular lesions composed of abundant dense collagen (16). But these MR imaging findings are not specific to desmoid tumors. In MRI of our patient, a similar imaging appearance on $\mathrm{T} 1$ and $\mathrm{T} 2$ weighted images was demonstrated as described above. Although it was not performed in our case, it has been reported that diffusionweighted imaging may help differentiate desmoid tumors from malignant soft tissue tumors, whereas the desmoid tumor demonstrates a higher mean apparent diffusion coefficient (ADC) than malignant soft tissue tumors (17). PET/CT imaging characteristics of desmoid tumor show varying FDG uptake, which probably reflects varying proportions of active cellular tissue and collagen in the lesion. The areas of high FDG metabolism are likely to represent more cellular and mitotically active areas $(2,18)$. In our patient, a moderate FDG tracer localization ( $\mathrm{SUV}_{\max }$ of 3.1) was seen in the tumor, suggesting that it has more biological aggressiveness and a tendency for recurrence than other desmoid tumors with a low level FDG uptake. Therefore, our patient should be evaluated carefully during the follow-up examinations.

In conclusion, chest wall desmoid tumor is a rare entity, which should be considered in the differential diagnosis of malignant local tumor recurrence after modified radical mastectomy. Failure to recognize it or an incorrect diagnosis as a postoperative change may lead to a delayed diagnosis and treatment like our patient. In breast cancer patients with a previous operation history, desmoid tumor is also a mimicking recurrent breast cancer or other soft tissue tumors of mesenchymal origin. Therefore, biopsy and histologic examinations are essential for avoiding misdiagnosis and proper management.

\section{Footnote}

Authors' Contribution: Yeong Yi An and Kyeong A Choi participated in conception of study, data collection and analysis and drafted the manuscript. All authors read and approved the final manuscript.

\section{References}

1. Erguvan-Dogan B, Dempsey PJ, Ayyar G, Gilcrease MZ. Primary desmoid tumor (extraabdominal fibromatosis) of the breast. $A J R$ Am J Roentgenol. 2005;185(2):488-9. doi: 10.2214/ajr.185.2.01850488. [PubMed: 16037525].

2. Souza FF, Fennessy FM, Yang Q, van den Abbeele AD. Case report. PET/CT appearance of desmoid tumour of the chest wall. Br J Radiol. 2010;83(986):39-42. doi: 10.1259/bjr/18648939. [PubMed: 20139256].

3. Lopez-Ruiz J, Ruiz M, Echevarria JJ, Lopez S, Imaz I. Mammary fibromatosis mimicking recurrent breast cancer: radiological findings. Eur Radiol. 2005;15(9):2034-6. doi: 10.1007/s00330-005-2717-x. [PubMed: 15806367].

4. Yeo W, Teo PML, Kwan WH, Suen M. Desmoid tumor mimicking local recurrence of carcinoma of the breast. Int J Clin Oncol. 1998;3(1):49-51. doi: 10.1007/bf02490103. 
5. Neuman HB, Brogi E, Ebrahim A, Brennan MF, Van Zee KJ. Desmoid tumors (fibromatoses) of the breast: a 25-year experience. Ann Surg Oncol. 2008;15(1):274-80. doi: 10.1245/s10434-007-9580-8. [PubMed: 17896146].

6. McKinnon JG, Neifeld JP, Kay S, Parker GA, Foster WC, Lawrence WJ. Management of desmoid tumors. Surg Gynecol Obstet. 1989;169(2):104-6. [PubMed: 2756458].

7. Brown CS, Jeffrey B, Korentager R, Hughes K. Desmoid tumors of the bilateral breasts in a patient without Gardner syndrome: a case report and review of literature. Ann Plast Surg. 2012;69(2):220-2. doi: 10.1097/SAP.ob013e31821e8faf. [PubMed: 21629058].

8. Lopez R, Kemalyan N, Moseley HS, Dennis D, Vetto RM. Problems in diagnosis and management of desmoid tumors. Am J Surg. 1990;159(5):450-3. [PubMed: 2139764].

9. Gergele F, Guy F, Collin F, Krause D. A desmoid tumour associated with a breast prosthesis. Diagn Interv Imaging. 2012;93(3):200-3. doi: 10.1016/j.diii.2011.12.011. [PubMed: 22421285].

10. Hammoudeh ZS, Darian VB. Desmoid tumor (fibromatosis) of the breast after augmentation with saline implants. Plast Reconstr Surg. 2012;129(4):753-4. doi: 10.1097/PRS.ob013e318245e918. [PubMed: 22456408].

11. Jeong WS, Oh TS, Sim HB, Eom JS. Desmoid tumor following augmentation mammoplasty with silicone implants. Arch Plast Surg. 2013;40(4):470-2. doi: 10.5999/aps.2013.40.4.470. [PubMed: 23898455].
12. Ha KY, Deleon P, Hamilton R. Breast fibromatosis mimicking breast carcinoma. Proc (Bayl Univ Med Cent). 2013;26(1):22-4. [PubMed: 23382604].

13. Glazebrook KN, Reynolds CA. Mammary fibromatosis. AJR Am J Roentgenol. 2009;193(3):856-60. doi: 10.2214/AJR.08.1892. [PubMed: 19696302].

14. Leibman AJ, Kossoff MB. Sonographic features of fibromatosis of the breast. J Ultrasound Med. 1991;10(1):43-5. [PubMed: 1997685].

15. O'Sullivan P, O'Dwyer H, Flint J, Munk PL, Muller N. Soft tissue tumours and mass-like lesions of the chest wall: a pictorial review of CT and MR findings. Br J Radiol. 2007;80(955):574-80. doi: 10.1259/bjr/16591964. [PubMed: 16728417].

16. Walker EA, Petscavage JM, Brian PL, Logie CI, Montini KM, Murphey MD. Imaging features of superficial and deep fibromatoses in the adult population. Sarcoma. 2012;2012:215810. doi: 10.1155/2012/215810. [PubMed: 22966216].

17. Oka K, Yakushiji T, Sato H, Fujimoto T, Hirai T, Yamashita Y, et al. Usefulness of diffusion-weighted imaging for differentiating between desmoid tumors and malignant soft tissue tumors. J Magn Reson Imaging. 2011;33(1):189-93. doi: 10.1002/jmri.22406. [PubMed: 21182138].

18. Basu S, Nair N, Banavali S. Uptake characteristics of fluorodeoxyglucose (FDG) in deep fibromatosis and abdominal desmoids: potential clinical role of FDG-PET in the management. Br J Radiol. 2007;80(957):750-6. doi: 10.1259/bjr/53719785. [PubMed: 17709361]. 